Sonderabdruck aus

SCHWEIZERISCHE ZEITSCHRIFT FUR HYDROLOGIE BIRKHÄUSER VERLAG BASEL

Vol. XVIII 1956 Fasc. 1 


\title{
Über die Entnahme von Wasserproben in fliessenden Gewässern
}

\author{
Von O. JAAG, H. AMBÜHL und P. ZIMMERMANN \\ (Mitteilung aus der Eidgenössischen Anstalt für Wasserversorgung, Abwasser- \\ reinigung und Gewässerschutz an der Eidgenössischen Technischen Hochschule, \\ Zürich) \\ Eingegangen am 6. Oktober 1955
}

Im Laufe unserer Untersuchungen über den Chemismus, insbesondere den Sauerstoff- und Kohlensäurehaushalt fliessender Gewässer, zeigte es sich, dass die üblichen Methoden der Probenahme in vielen Fällen dem besonderen Zweck der Aufgabe nicht genügen. So erfordert besonders das Abfüllen der Sauerstoff-Probeflaschen grosse Sorgfalt, die mit der herkömmlichen Arbeitsweise (Schöpfbecher, Einfüllen des Wassers durch Einlaufenlassen an der Wasseroberfläche und dergleichen) nicht zu erreichen ist. Besonders an Stellen, wo es darauf ankommt, geringe Unterschiede sicher nachzuweisen, sind wir darauf angewiesen, die bei der Probenahme unvermeidlichen Ungenauigkeiten möglichst zu vermindern:

\section{1. "Horizontal-Schöpfflasche»}

(Abbildung 1)

Zweckdienliche Voruntersuchungen führten uns dazu, ein kleines, handliches Gerät zu entwickeln, das gestattet, Wasser an jedem beliebigen Punkt eines Gewässers zu entnehmen und auf die übliche korrekte Weise (längeres Durchlaufenlassen durch die Probeflasche) abzufüllen.

Es handelt sich dabei um einen Apparat, der in gewissen Einzelheiten der Friedingerschen Schöpfflasche für Seeuntersuchungen nachgebildet ist (Abb. I). In Normalstellung werden die beiden Deckel durch Spiralfedern offengehalten. Zur Probenahme wird die an einer Stange befestigte Flasche derart in die Strömung gehalten, dass das Wasser frei durchziehen kann. Nach ungefähr einer Minute (Temperaturausgleich!) schliesst man die 
Deckel durch Zug an zwei Kabeln. Zwei besondere Klinken verhindern, dass sich die Flasche unbeabsichtigterweise wieder öffnen kann.

Das Gerät ist ganz aus Antikorodal angefertigt. Dieses Leichtmetall hat trotz grosser mechanischer Festigkeit den Vorteil, dass einerseits das Gewicht auf ein Minimum gebracht werden kann und dass anderseits keinerlei Korrosion (wichtig für chemische Untersuchungen!) eintritt. Die heutige Schweiss- und Lötetechnik für Leichtmetalle ist hinreichend entwickelt, so dass die Herstellung in der Werkstatt keine grossen Schwierigkeiten bereitet.

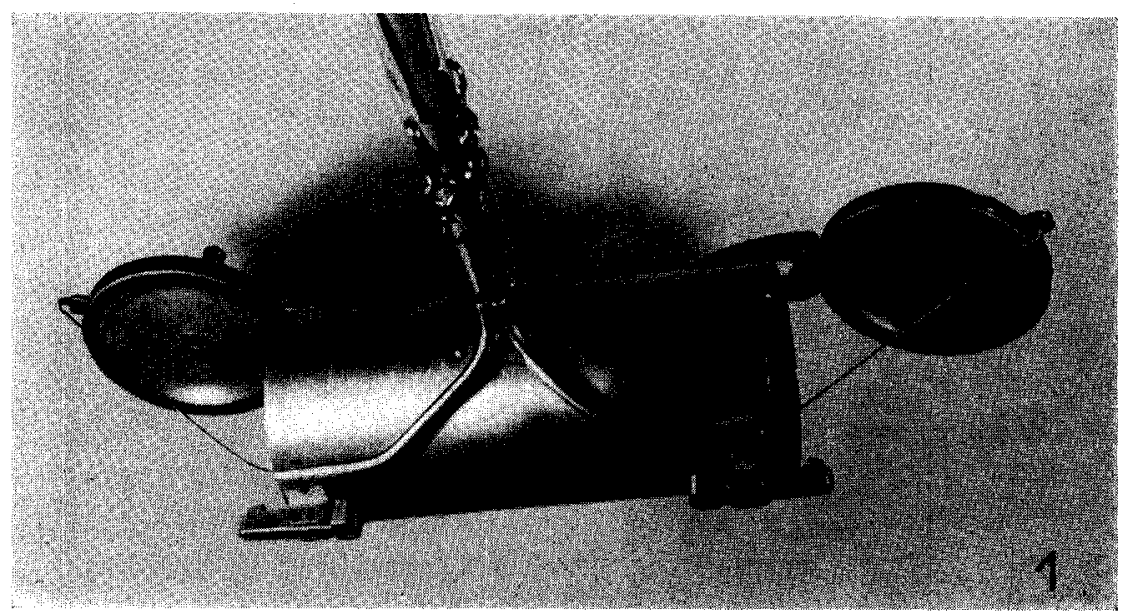

Abb. 1. "Horizontal-Schöpfflasche» in Arbeitsstellung mit geöffneten Deckeln.

Das Volumen ist vorderhand auf $1 / 2$ und I 1 beschränkt, doch befindet sich ein grösseres, 21 fassendes Modell in Vorbereitung.

Die Horizontal-Schöpfflasche hat sich in längerem und zeitweise sehr strengem Gebrauch bestens bewährt. Allein der Umstand, dass die Probenahme an genau definierten Stellen - in der Regel in einem bestimmten Abstand von der Gewässersohle, meistens jedoch nicht an der Oberfläche vorgenommen werden kann, dürfte für eine weite Verbreitung angezeigt sein. Dazu macht sich besonders bei Reihenuntersuchungen, die in kurzer Zeit durchgeführt werden müssen, die bequeme Handhabung angenehm bemerkbar.

Als Zubehör zur Schöpfflasche wie auch zu sämtlichen übrigen Sammelgeräten verwenden wir seit rund zwei Jahren einen Satz von je $40 \mathrm{~cm}$ langen, mit Messinggewinden versehenen Antikorodalrohren. Diese Rohr- 
stücke von $20 \mathrm{~mm}$ Durchmesser können ohne weiteres im Rucksack mitgeführt werden, sind leicht, sehr stabil und lassen sich auf jede beliebige Länge zusammenstellen.

Für die mikrochemische Untersuchung stagnierender Zonen hinter grösseren Erhebungen im Bachbett (Zonen hinter grossen Steinen, Kolke usw.) bauten wir zwei besondere Geräte, die eine einwandfreie Entnahme der Wasserproben ermöglichen. Dabei mussten die Fassungsvolumina ausserordentlich klein gehalten werden, da einer Ruhezone, beispielsweise im «Strömungsschatten» eines grossen Steins, nur wenig Wasser entnommen werden darf. Durch die Entnahme fliesst natürlich eine entsprechende Menge Wasser nach und führt zu einer Vermischung bzw. Verdünnung, die um so grösser ist, je mehr Wasser entnommen wird.

\section{2. "Mikro-Schöpfflasche»}

(Abbildungen 2 und 3 )

Dieses Gerät ist im wesentlichen die stark verkleinerte Ausführung der oben beschriebenen «Horizontal-Schöpfflasche». Dank ihrer kleinen Abmessungen hat sie wesentlich geringeren Kräften standzuhalten, weshalb der Verschlussmechanismus der Deckel etwas anders gestaltet wurde:

Nocken und Blattfeder fixieren die Deckel im geöffneten Zustande. Durch einen Kabelzug können sie geschlossen werden, worauf sie durch den erwähnten Mechanismus angepresst werden, indem der Totpunkt in der Mitte zwischen beiden Stellungen (geöffnet, geschlossen) liegt.

Der Auslaufstutzen besitzt einen inneren Durchmesser von $4 \mathrm{~mm}$. Unter der Voraussetzung, dass beide Deckel hermetisch verschliessen, kann man auf das Anbringen eines Hahns verzichten. Es hat sich jedenfalls gezeigt, dass auch ohne Hahn kein Wasser aus der vollständig gefüllten Schöpfflasche entweicht, sofern die Lichtweite des ungefähr I,5 cm langen Auslaufröhrchens klein dimensioniert und mit dem Gerät ruhig manipuliert wird. Um das gefasste Wasser abzufüllen, braucht man bloss den oberen Deckel leicht zu öffnen, damit Luft nachströmen kann.

Die Wassermenge, die auf einmal gefasst werden kann $\left(40 \mathrm{~cm}^{3}\right)$, reicht für eine kolorimetrische Bestimmung, zum Beispiel von Ammoniak, von Nitrit, Nitrat oder Phosphat völlig aus. Auch für die Sauerstoffbestimmung genügt das Volumen bei weitem, sofern man entsprechende kleine Probefläschchen verwendet. Bei unseren Arbeiten haben sich seit längerem kleine, bloss $30-35 \mathrm{~cm}^{3}$ fassende Fläschchen mit keilförmig abgeschliffenen Stopfen bestens bewährt. Die Genauigkeit der Bestimmung (geschickte 
Manipulation, vor allem sehr rasche und sichere Titration aus der Mikrobürette oder -pipette vorausgesetzt) leidet kaum; jedenfalls ergaben Reihenversuche zwischen den beschriebenen kleinen Fläschchen und den üblichen grossen, $250 \mathrm{~cm}^{3}$ fassenden Probeflaschen keine wesentlichen Streuungsunterschiede. Die Vorteile einer solchen Reduktion des Flascheninhalts sind ja leicht einzusehen: Felduntersuchungen beispielsweise, die infolge schlechter Wegverhältnisse die Verwendung eines Fahrzeuges aus-
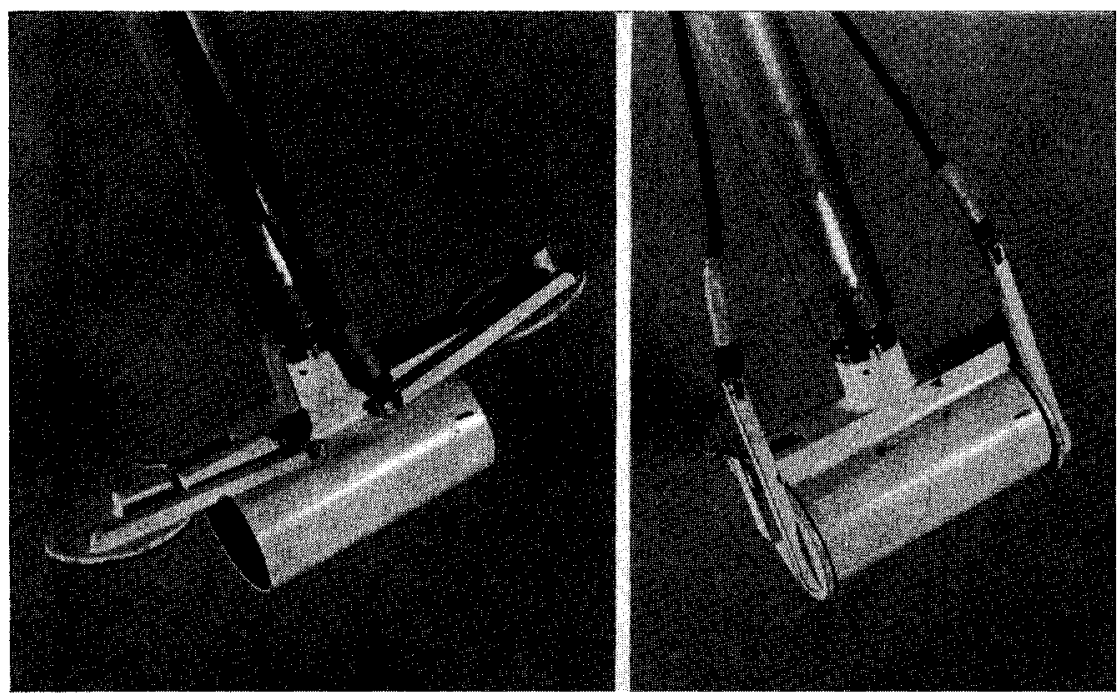

Abb. 2. "Mikro-Schöpfflasche» mit geöffneten Deckeln.

Abb. 3. "Mikro-Schöpfflasche», Deckel geschlossen. Die beidseits herausragende Blattfeder ist gut sichtbar.

schliessen, können nichtsdestoweniger mit der gleichen Präzision und Dichte der Probestellen durchgeführt werden wie im Idealfall, ein Umstand, der allein schon die Anwendung kleinerer Volumina für die Sauerstoffbestimmung rechtfertigen dürfte, von Gebirgsuntersuchungen mit ihrem beschwerlichen Materialtransport ganz zu schweigen.

\section{3. "Probenahmestab"}

(Abbildung 4)

Dieselben Überlegungen, die zum Bau der «Mikroschöpfflasche» führten, bewogen uns, noch ein zweites Gerät zu entwickeln, das die Entnahme kleiner Wasserproben auch an sehr unzugänglichen und genau bestimmten Stellen gestattet. 
Es handelt sich um ein stabförmiges Ventil von I2 mm äusserem Durchmesser und etwa Io $\mathrm{cm}$ Länge, das durch eine Druckfeder geschlossen bleibt. Am hinteren Ende kann eine Probeflasche (kleine Sauerstofflasche) aufgesetzt werden, worauf das ganze System entweder sofort verwendet oder je nach Bedarf mit Stickstoff oder Kohlensäure durchgespült wird, um speziell bei Sauerstoff bestimmungen eventuelle Fehler auszuschliessen.

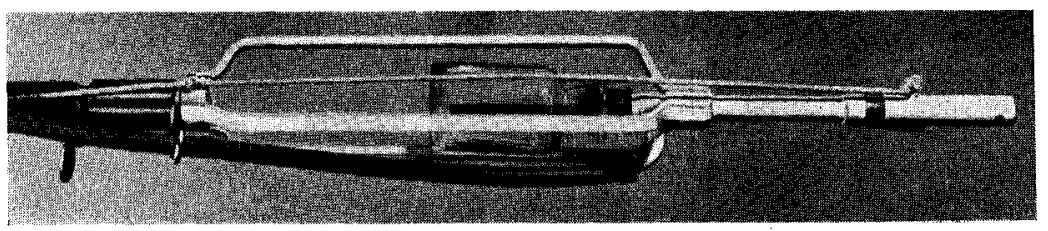

Abb. 4. "Probenahmestab" mit aufgesetzter Probeflasche.

Der Apparat ist über ein Gelenk mit der vorgängig beschriebenen Aluminiumstange zu einer Einheit verbunden. Das Gelenkstück gestattet, den «Probenahmestab» in jeder beliebigen Lage zu fixieren, so dass praktisch jeder «Schlupfwinkel» erreichbar ist.

Zur Probenahme wird das Gerät sorgfältig an die Probestelle bewegt und durch Zug an einem Kabel das Ventil geöffnet, worauf das Wasser einströmt. Die dabei verdrängte Luft bzw. vorher eingefüllter Stickstoff oder Kohlensäure entweicht durch ein eingelötetes, bis an den Flaschenboden reichendes Röhrchen, das durch einen dünnen Schlauch bis ans hintere Ende der Haltestange weitergeführt ist. Dadurch füllt sich das Fläschchen in 2-3 Sekunden, sofern man nicht in Nähe der Wasseroberfläche zu arbeiten hat. Die auf einmal gefasste Wassermenge reicht aus für eine Sauerstoff bestimmung oder für kolorimetrische Analysen, die nicht mehr als $30-35 \mathrm{~cm}^{3}$ Wasser benötigen. 